\title{
The Possible Role of A Mechanical Engineer in Biomedical Sciences
}

\author{
Mehmet Bozdoğanoglu ${ }^{1}$ and Ethem Unal ${ }^{2 *}$ \\ ${ }^{1}$ Department of Mechanical Engineering, Turkey \\ ${ }^{2}$ Department of General Surgery, Turkey
}

*Corresponding author: Ethem Unal, Health Sciences University, Umraniye Training and Research Hospital, Department of General Surgery, Istanbul, Turkey

Submission: 眥 October 30, 2017; Published: 眥 November 30, 2017

\section{Opinion}

Mechanical engineers create solutions and solve problems, playing a central role in the design and implementation of moving parts in a range of industries. Mechanical engineers provide efficient solutions to the development of processes and products, ranging from small component designs to extremely large plant, machinery or vehicles. They can work on all stages of a product, from research and development to design and manufacture, through to installation and final commissioning. Most industries rely on a form of mechanical systems and mechanical engineering is thought to be one of the most diverse of all engineering disciplines. Due to this, there are employment opportunities in a range of sectors, including: aerospace, automotive, biomedical, construction and manufacturing. Mechanical engineers can be involved in the management of people, projects and resources, as well as the development and use of new materials and technologies.

Medical engineers apply engineering principles to research and development of medical applications, treatments or diagnostic technologies related to acute or chronic medical conditions [1]. Medical engineers are employed by research organizations, manufacturers, government agencies or major medical centers.

A mechanical engineer specialized in medical science can be called as 'medical engineer'. Engineer's responsibilities in medicine can include research, development, testing and evaluation of medical devices, advisement on new biomedical purchases for hospitals and medical centers [2]. Medical engineers may specialize in medical devices, sports medicine, stem cell research, biomarkers, genomics, nanotechnology and more. Medical engineering projects can include artificial organs and prosthetic limbs, computerassisted procedures and treatments, informatics and medical imaging.

Medical engineers typically need a bachelor's degree in engineering or physics, chemistry or biology for any kind of entrylevel job. Most medical engineering jobs require a master's degree or Ph.D. in medical engineering. Master's degree programs can take up to two years and Ph.D. study can take up to seven years beyond the 4-year bachelor's degree program. Medical engineers often study technology, medical devices and diagnostics, computer modeling, analysis, physiology, biology, chemistry and physics. Medical engineers also usually complete a course of clinical study as well.

\section{References}

1. Raman R, Mitchell M, Perez-Pinera P, Rashid B, Lizanne D, et al. (2016) Design and integration of a problem-based biofabrication course into an undergraduate biomedical engineering curriculum. J Biol Eng 10: 10.

2. Eberhardt AW, Johnson OL, Kirkland WB, Dobbs JH, Moradi LG, et al. (2016) Team-Based Development of Medical Devices: An EngineeringBusiness Collaborative. J Biomech Eng 138(7): 31-35. 